\title{
Hydrodynamic Instabilities and Surface Waves in a Flow over an Impedance Wall
}

\author{
S.W. Rienstra \\ Nationa1 Aerospace Laboratory NLR \\ P.0. Box 90502, 1006 BM Amsterdam
}

\section{Abstract}

A 2-D analysis is made of the surface waves in a subsonic compressible uniform mean flow along an impedance wall. It is found that a maximum of four surface waves is possible. The regions of existence in complex impedance and wave number planes are given. One wave is found to travel upstream, while the others, one of which is an instability, propagate downstream. A WienerHopf solution of plane wave scattering at a hard/soft discontinuity of the wall impedance is constructed, in which the instability wave provides the additional degree of freedom to satisfy the Kutta condition at the impedance jump. The present waves are limiting forms of certain irregular acoustic duct modes.

\section{Introduction}

It was recognized in Morse and Ingard [1] that 2 of the acoustic modes in a duct with an impedance wall sometimes show a certain irregular behaviour. In the presence of mean flow through the duct 2 additional modes appear (Tester [2], Koch and Möhring [3], Eversman [4]), also of unusual character since one of them is to be considered as an instability. A 2-D/semi-infinite analysis is described in the present paper to investigate these modes further; this is possible because these modes are not essentially duct modes, but only coupled to the impedance wall. So, the present analysis embodies a high-frequency limit of the duct problem. The present 2-D configuration allows more explicit and transparant results, revealing better the essential physics.

\section{Mode1}

Consider a perturbation potential $\phi$ and pressure $p$ (dimensionless with mean density $\rho_{0}$, mean sound speed $c_{0}$, and some length) on a uniform mean flow with velocity $U_{0}=M c_{0}(0<M<1)$ in the space $y \geqq 0,-\infty<x<\infty$, satisfying

$$
\begin{aligned}
& \nabla_{\phi}^{2}-\left(i \omega+M \partial_{x}\right)^{2} \phi=0 \\
& p=-\left(i \omega+M \partial_{x}\right) \phi
\end{aligned}
$$


where the time harmonic factor $\exp (i \omega t)$ is suppressed throughout. The boundary condition at $y=0$ describes the effect of the complex impedance $z$

(Re $\mathrm{Z} \geqq 0$ ) modified by the (vanishing) mean flow boundary layer (Ingard [5]):

$$
\left(i \omega+M \partial_{x}\right)^{2} \phi=i \omega Z \partial_{y} \phi \quad, \quad y=0
$$

Furthermore:

$$
\phi \rightarrow 0 \quad, \quad y \rightarrow \infty
$$

\section{Surface waves}

Consider waves of the form

$$
\phi=\exp (-i \alpha x-i \gamma y)
$$

With the transformation $\omega=\beta \Omega, \sigma=\beta \alpha+M \Omega, \beta=\left(1-M^{2}\right)^{\frac{1}{2}}$, (1) and (2) yield

$$
\begin{aligned}
& \gamma(\sigma)=\left(\Omega^{2}-\sigma^{2}\right)^{\frac{1}{2}} \\
& K(\sigma)=\beta^{3} \Omega Z_{\gamma}+(\Omega-M \sigma)^{2}=0
\end{aligned}
$$

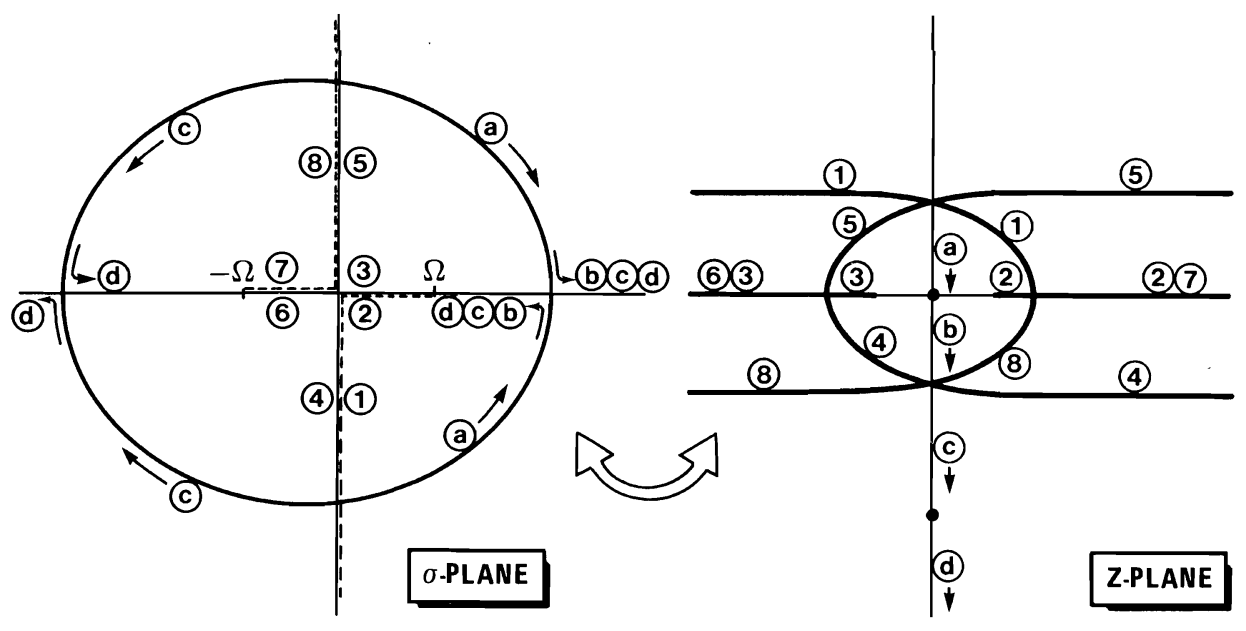

Fig. 1 Mapping $Z=-(\Omega-M \sigma)^{2} / \beta^{3} \Omega \gamma$

$$
\text { (1), }, \ldots \text {, (d) from } \sigma \text { from } Z \text { to } \sigma
$$


The L-shaped branch cuts of $\gamma$ along the lines $\operatorname{Im} \gamma=0$, with the choice of the branch with Im $\gamma \leqq 0$, garantees (3) (Fig. 3). The -at most 4- solutions $\sigma$ of (4) correspond to the waves under consideration. Instead of solving $\sigma$, we start with considering equation (4) as defining $\mathrm{Z}=\mathrm{Z}(\sigma)$. Then, by mapping the branch cuts of $\gamma(\sigma)$, which are "lines of disappearance" of solutions $\sigma$, from the $\sigma-p l a n e$ to the $Z$-plane (Fig. 1) we obtain regions $I$ to $V$ in $Z$ with different number of solutions of (4). Mapping back from $Z$ to $\sigma$ the line $\operatorname{Re} Z=0$ we obtain in the $\sigma$-plane the "egg-1ike" contour displaying the regions of existence of the various solutions. See figures 2 and 3 . Note that the finer division II, III, IV was missed by previous authors ([4], [3]). For further details, see appendix A. From figures 2 and 3 it is seen that if $M \rightarrow o, \omega$ fixed (no-flow limit), two solutions disappear to infinity, while the division of the $Z$-plane is only into two parts, along Im $Z=0$, which is in accordance with Wenzel [6]. Another interesting limit is $M \rightarrow 0, \omega \rightarrow 0$, with $S=\omega / M$ (Strouhal number) fixed (incompressible limit). If $Z^{\prime}(=Z / M)$ is the impedance dimensionless on $\rho_{0} U_{0}$, the solutions $\sigma$ (if existing) are explicitly given by

$\sigma=i S\left(\frac{1}{2} Z^{\prime}-i \pm\left(1+\left(\frac{1}{2} Z^{\prime}-i\right)^{2}\right)^{\frac{1}{2}}\right),-i S\left(\frac{1}{2} Z^{\prime}+i \pm\left(1+\left(\frac{1}{2} Z^{\prime}+i\right)^{2}\right)^{\frac{1}{2}}\right) .(5)$

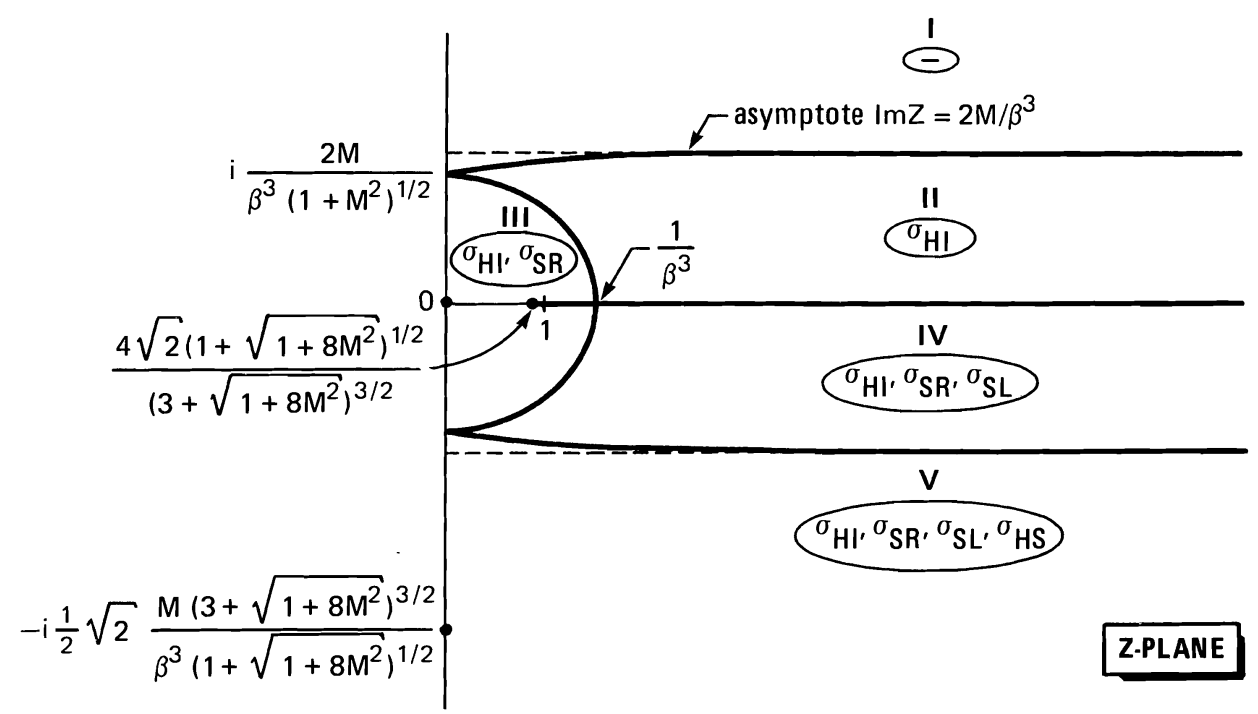

Fig. 2 Complex impedance $\mathrm{Z}$-plane, divided into the regions with different number of solutions $\sigma$ 


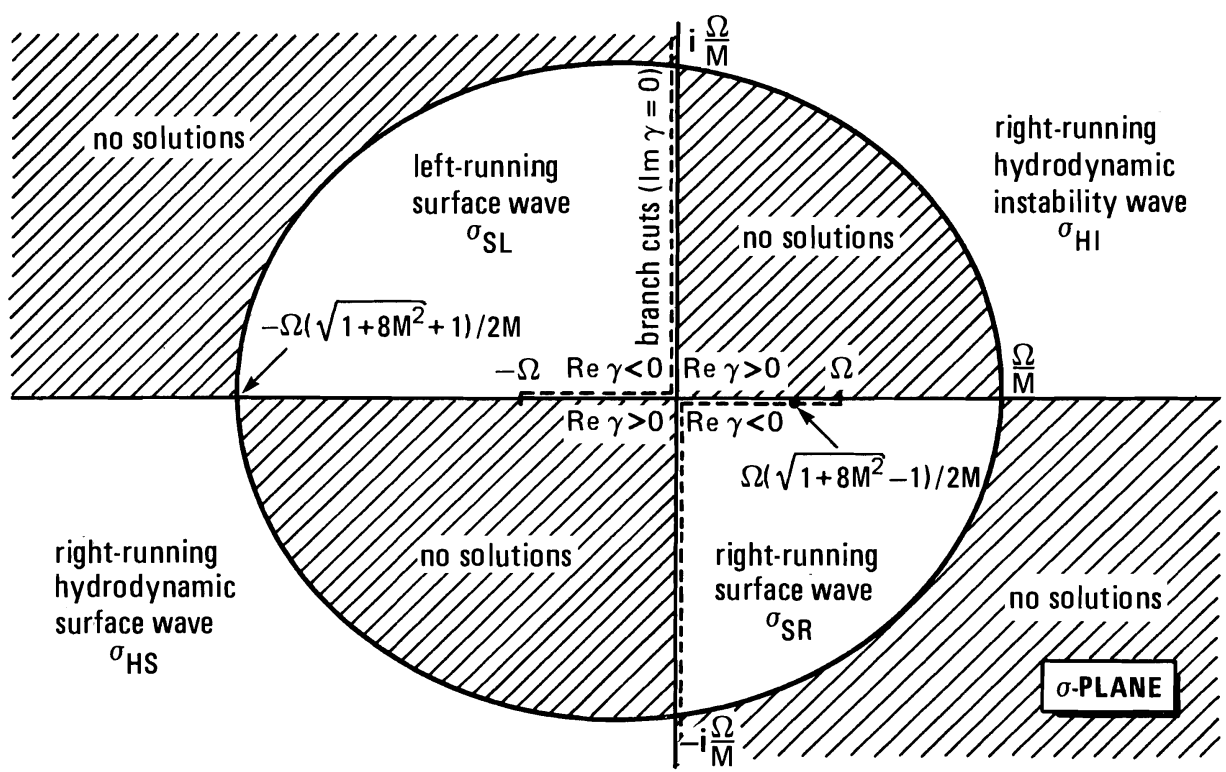

Fig. 3 Complex (transformed) wave number $\sigma-p l a n e$, with regions of existence of the surface waves

Furthermore, $\gamma(\sigma)=-i \operatorname{sgn}(\operatorname{Re} \sigma) \sigma$, the contour in the $\sigma$-plane becomes a circle with radius $S$, and the division of the $Z^{\prime}-p l a n e$ becomes threefold, viz. I: $\operatorname{Im} Z^{\prime}>2$, III: $-2<\operatorname{Im} Z^{\prime}<2$, V: $\operatorname{Im} Z^{\prime}<-2$.

\section{Scattering problem}

The scattered field generated by the plane waves

$$
p=\frac{1}{2} \exp \left(-i \Omega\left(\cos \theta_{s}-M\right) x-i \Omega \sin \Theta_{s} y\right)
$$

$\left(\mathrm{x}=\beta \mathrm{X},-\pi<\theta_{\mathrm{S}}<0\right)$, incident to a wall $\mathrm{y}=0$, hard along $\mathrm{x}<0$, and soft $(\rightarrow(2)$ ) along $x>$ o (Fig. 4), is found by a Wiener-Hopf procedure ([7], [3]):

$$
\begin{aligned}
& \mathrm{p}=\exp \left(-\mathrm{i} \Omega\left(\cos \Theta_{\mathrm{s}}-\mathrm{M}\right) \mathrm{X}\right) \cos \left(\Omega \sin \Theta_{\mathrm{s}} \mathrm{y}\right) \\
& +\frac{1}{2 \pi i} \oint_{-\infty}^{\infty}\left\{\frac{\mathrm{K}_{-}\left(\Omega_{1}\right) \gamma_{-}\left(\Omega_{1}\right)}{\sigma-\Omega_{1}}+\mathrm{E}\right\} \frac{(\Omega-\mathrm{M} \sigma)^{2}}{\mathrm{~K}_{+}(\sigma) \gamma_{-}(\sigma)} \exp (-\mathrm{i} \gamma \mathrm{y}-\mathrm{i} \alpha \mathrm{x}) \mathrm{d} \sigma
\end{aligned}
$$


where $\mathrm{K}(\sigma)=\mathrm{K}_{+}(\sigma) / \mathrm{K}_{-}(\sigma), \gamma_{ \pm}(\sigma)=(\Omega \mp \sigma)^{\frac{1}{2}}, \Omega_{1}=\Omega \cos \Theta_{\mathrm{s}}$, and $\mathrm{E}$ a constant (the amplitude of an eigensolution). See appendix B. Apart from an upward identation of the integration contour around $\Omega_{1}$, the location of the contour around the poles, and thereby the split functions $K_{ \pm}$, is not readily evident. The causality argument of Jones and Morgan [8] (a refinement of Briggs [9]) tells us to construct the correct solution by analytic continuation in $\omega$ from negative imaginary $(\omega=-i|\omega|)$ to real values. This requires to adopt some frequency dependent $Z=Z(\omega)$, to deal with a physically realizable impedance. For the moment we have chosen a mass-spring-damper system: $z=r+i m \omega-i k / \omega$.

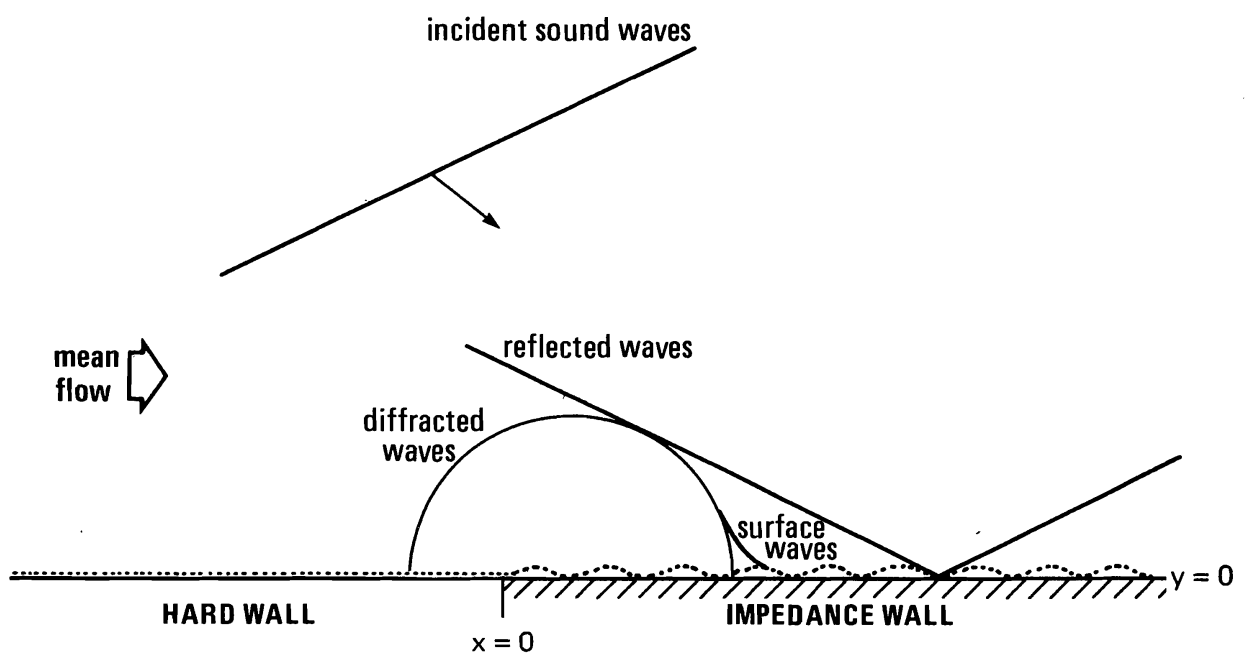

Fig. 4 Plane sound waves in an uniform mean flow, scattered by a wall impedance discontinuity

One zero of $\mathrm{K}$, namely $\sigma_{\mathrm{HI}}$ (Fig. 3), appears for $\omega=-i|\omega|$ to move to the lower complex half-plane. For the general case a numerical search gave the evidence, but for the incompressible case (5) it can be proved analytically (App. C). As a consequence, $\mathrm{K}_{+}(\sigma)$ contains the factor $\sigma-\sigma_{H I}$, and for real $\omega$ the contour has to be deformed such that it remains "above" the pole $\sigma_{H I}$ (Fig. 5); thus $\sigma_{H I}$ corresponds to a right-running instability. If $z \in I$ (for real $\omega) \sigma_{H I}$ is in the second quadrant on the other Riemann sheet of $\gamma$, and the "causal" contour yields an unacceptable solution unbounded in $y$. In this case it is probably preferable to avoid the deformation; $k_{+}$contains no fac- 


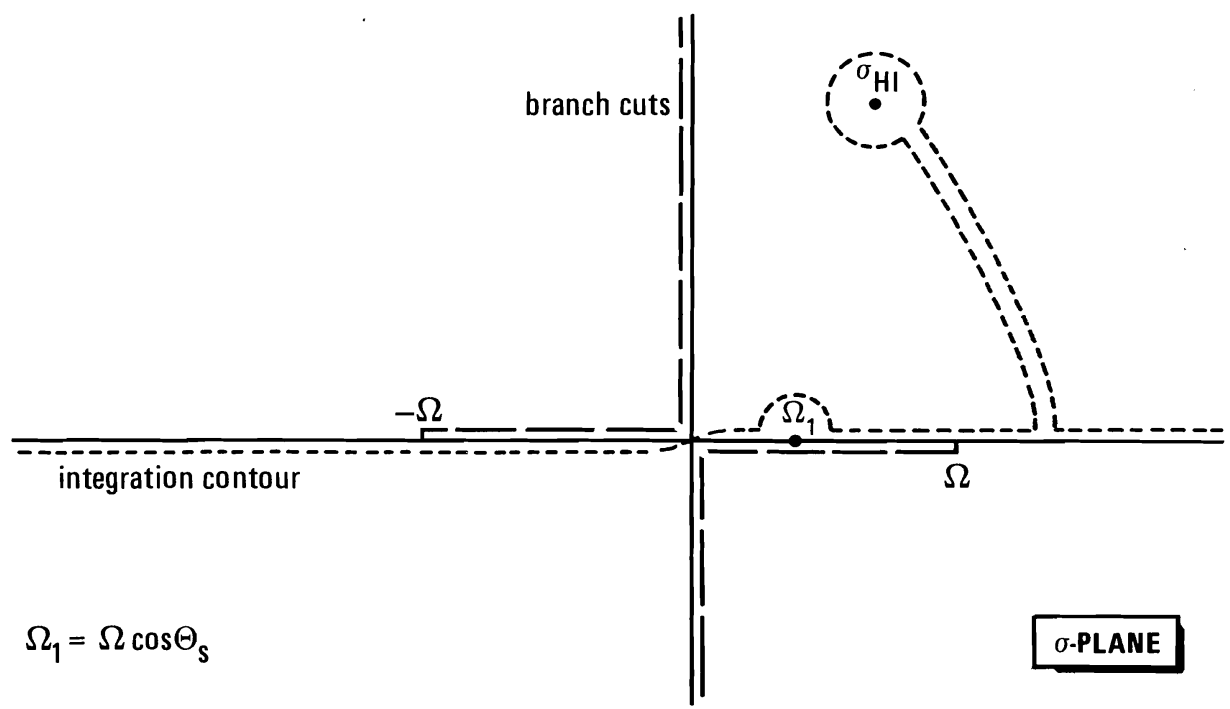

Fig. 5 Integration contour in $\sigma-p l a n e$

tor $\sigma-\sigma_{\mathrm{HI}}$, and $\mathrm{E}=\mathrm{o}$. If $\mathrm{Z} \notin \mathrm{I}$, the available eigensolution with amplitude E provides the possibility to satisfy the Kutta condition at $\mathrm{x}=\mathrm{y}=0$, with the choice $\mathrm{E}=\mathrm{o}$. In this case the sound couples to the generated surface waves in a way that acoustic energy may be exchanged with hydrodynamic (kinetic) energy, as was shown by Quinn and Howe [10] for the special case $Z=0$, where $K$ can be split by inspection $\left(K_{+}=K, K_{-}=1\right)$, and (6) can be reduced to Fresnel's integrals.

\section{Relevance to duct modes}

The axial wave numbers of the modes in a cylindrical duct of radius unity are given by

$$
\left(\Omega-M_{\sigma}\right)^{2}-i \beta^{3} \Omega Z_{\gamma} J_{m}^{\prime}(\gamma) / J_{m}(\gamma)=0
$$

which is the analogue of equation (4). $\mathrm{J}_{\mathrm{m}}$ denotes the $\mathrm{m}-\mathrm{th}$ order Bessel function. In the high frequency limit $\Omega \rightarrow \infty$ we can distinguish two cases: Im $\gamma=O(\Omega)$ in which case eq. (7) reduces to eq. (4), and $\operatorname{Im} \gamma=O(1)$ which amounts to a boundary layer in the $\sigma$-plane enclosing the branch cuts of $\gamma$. The solutions inside this boundary layer correspond to genuine duct modes (their field covering the whole duct cross-section. The other possible four 
(or two, if $M=0$ ) with $\operatorname{Im} \gamma=O(\Omega)$ are surface waves, asymptotically described by the above analysis. If $\mathrm{Z}$ varies from one region to another, e.g. from I to II (Fig. 2), one of the genuine duct modes changes its character and turns into a surface wave, which is, being confined to the wall region, a physically different phenomenon.

\section{References}

1. Morse, P.M.; Ingard, K.U.: Theoretical Acoustics. McGraw-Hil1, 1968 (509).

2. Tester, B.J.: JSV 28(2) (1973) 151-203.

3. Koch, W.; Möhring, W.: AIAA Jn1 21(2) (1983) 200-213.

4. Eversman, W.: JSV 50(1) (1977) 159-162.

5. Ingard, K.U.: JASA 31(7) (1959) 1035-1036.

6. Wenze1, A.R.: JASA 55(5)(1974) 956-963.

7. Noble, B.: The Wiener-Hopf Technique, Pergamon 1958.

8. Jones, D.S.; Morgan, J.D.: Proc. Roy. Soc. L. A 338 (1974) 17-41.

9. Briggs, R.J.: Res. Monogr. No. 29 MIT 1964.

10. Quinn, M.C.; Howe, M.S.: JSV 97 (1)(1984) 1-9.

\section{APPENDIX A}

See figure 2. Impedances of special interest are $\left(\right.$ introduce $\left.w=\left(1+8 M^{2}\right)^{\frac{1}{2}}\right)$ :

$$
\begin{aligned}
& Z=2 i M / \beta^{3}\left(1+M^{2}\right)^{\frac{1}{2}} \quad \text { where } \quad \sigma_{H I}=i \Omega / M \quad, \quad \sigma_{S R}=-i \Omega / M \text {. } \\
& Z=-2 i M / \beta^{3}\left(1+M^{2}\right)^{\frac{1}{2}} \quad \text { where } \quad \sigma_{S L}=i \Omega / M \quad, \quad \sigma_{H S}=-i \Omega / M \text {. } \\
& Z=1 / \beta^{3} \quad \text { where (in the upper half of III) } \sigma_{S R}=0 \text {, or (in IV) } \sigma_{S L}=0 \\
& \mathrm{Z}=\mathrm{o} \quad \text { where } \sigma_{\mathrm{HI}} \text { and } \sigma_{\mathrm{SR}} \text { coincide: } \sigma_{\mathrm{HI}}=\sigma_{\mathrm{SR}}=\Omega / \mathrm{M} \\
& Z=-i \frac{1}{2} \sqrt{2} M(3+w)^{3 / 2} / \beta^{3}(1+w)^{\frac{1}{2}} \text { where } \sigma_{H S} \text { and } \sigma_{S L} \text { coincide: } \\
& \sigma_{\mathrm{HS}}=\sigma_{\mathrm{SL}}=-\Omega(\mathrm{w}+1) / 2 \mathrm{M}
\end{aligned}
$$

When $Z$, varying in region III, crosses the real interval $\left(4 \sqrt{2}(1+w)^{\frac{1}{2}} /(3+w)^{3 / 2}, 1 / \beta^{3}\right)$ with, say, increasing imaginary part, then in the $\sigma-p l a n e \sigma_{\mathrm{SR}}$ disappears somewhere along $(\mathrm{o}, \Omega(\mathrm{w}-1) / 2 \mathrm{M})$, but appears at the same time somewhere along $(\Omega(\mathrm{w}-1) / 2 \mathrm{M}, \Omega)$, thus leaving the number of solutions the same. When $Z \rightarrow 4 \sqrt{ } 2(1+w)^{\frac{1}{2}} /(3+w)^{3 / 2}$ the two representations of $\sigma_{\mathrm{SR}}$ coincide (K becomes quadratic) at $\sigma_{\mathrm{SR}}=\Omega(\mathrm{w}-1) / 2 \mathrm{M}$.

If $\operatorname{Re} Z=0$ we have (see Fig.1) along (a) two complex conjugate solutions, which become real along (b); along (c) we have two complex conjugate and two real solutions, and along (d) all four are real. 


\section{APPENDIX B}

It is convenient to introduce the position of the wall streamline $y=h(x) \exp (i \omega t)$, and to write instead of (2) ([3],[5]): $\partial_{y} \phi=\left(i \omega+M \partial_{x}\right) h, \quad p=-i \omega Z h$ for $y=0, x>0$. Then, if

$$
\begin{aligned}
& \phi=\psi+\frac{i \beta \cos \left(\Omega \sin \theta_{s} y\right)}{\Omega\left(1-M \cos \theta_{s}\right)} \exp \left(-i \Omega\left(\cos \theta_{s}-M\right) x\right) \\
& \hat{\psi}(\alpha, y)=\int_{-\infty}^{\infty} \psi(x, y) \exp (i \alpha x) d x \quad, \quad H_{+}(\alpha)=\int_{0}^{\infty} h(x) \exp (i \alpha x) d x \\
& P_{-}(\alpha)=\int_{-\infty}^{0}\left(i \omega+M \partial_{x}\right) \psi(x, 0) \exp (i \alpha x) d x
\end{aligned}
$$

we obtain (assume for the moment $\cos \theta_{S}>0, \operatorname{Im} \Omega<0$ )

$$
\begin{aligned}
& \hat{\psi}=\mathrm{A} \exp (-i \gamma y), \quad A=-(\Omega-\mathrm{M \sigma}) \mathrm{H}_{+} / \beta \gamma, \\
& \mathrm{P}_{-}+i \beta /\left(\sigma-\Omega_{1}\right)=-i \mathrm{KH}_{+} / \beta^{2} \gamma .
\end{aligned}
$$

In the usual way ([7]) we rewrite this equation into a left hand side, analytic in $\sigma$ in a lower half plane (-), and a right hand side, analytic in an upper half plane $(+)$, both then equal to one entire function $E(\sigma)$. As explained in the main text, we start for causality with $\omega=-i|\omega|$. Then $\sigma_{H I}$ belongs to the lower half plane, and we have the estimates for $|\sigma| \rightarrow \infty$ : $\mathrm{K}_{+}(\sigma)=O\left(\sigma^{2}\right), \mathrm{K}_{-}(\sigma)=O(1)$. In addition, $\mathrm{H}_{+}(\sigma)=O\left(\sigma^{-3 / 2}\right), \mathrm{P}_{-}(\sigma)=O\left(\sigma^{-\frac{1}{2}}\right)$, as results from $h(x)=O\left(x^{\frac{1}{2}}\right), p(x, 0)=O\left(x^{-\frac{1}{2}}\right)$ for $x+o$. It turns out then that $\mathrm{E}$ is a constant. Collecting the various terms, a Fourier transform back into the $\mathrm{x}$-domain, and rotating $\omega$ from the negative imaginary axis to the positive real axis (preserving analyticity in $\omega$ by deforming the $\sigma$-integration contour, as explained in the main text) then gives eq. (6).

\section{APPENDIX C}

Since $\quad \sigma_{\mathrm{HI}}=i S \exp \left(\operatorname{arsinh}\left(\frac{1}{2} \mathrm{Z}^{\prime}-i\right)\right)$ we have $\arg \sigma_{H I}=\frac{1}{2} \pi+\arg S+\operatorname{Im}\left(\operatorname{arsinh}\left(\frac{1}{2} Z^{\prime}-i\right)\right)$. If $S \rightarrow-i|S|, Z^{\prime}$ becomes real and $\operatorname{Im}\left(\operatorname{arsinh}\left(\frac{1}{2} Z^{\prime}-i\right)\right)<0$, and hence $\arg \sigma_{H I} \in\left(-\frac{1}{2} \pi, 0\right)$. 